Bundesgesundheitsbl 2016 $\cdot 59: 1020-1027$

DOI 10.1007/s00103-016-2387-7

(C) Springer-Verlag Berlin Heidelberg 2016
Bekanntmachung des Umweltbundesamtes

\section{Aktualisierung der Referenzwerte für polychlorierte Biphenyle (PCB) im Blut}

\author{
Stellungnahme der Kommission Human-Biomonitoring \\ des Umweltbundesamtes
}

Aufgrund zahlreicher Hinweise, dass die PCB (polychlorierte Biphenyle)-Belastung der Bevölkerung in den letzten Jahren deutlich gesunken ist und dass die in den Jahren 1998 und 2003 publizierten Referenzwerte [1,2] nicht mehr gültig sein dürften, entschied sich die Kommission Human-Biomonitoring (HBM-Kommision), die Referenzwerte $\left(\mathrm{RV}_{95}\right)$ für $\mathrm{PCB}$ im Blut zu aktualisieren, um eine Bewertung heutiger Biomonitoringergebnisse $\mathrm{zu}$ ermöglichen.

Für die PCB zeigt eine Betrachtung der zeitlichen Entwicklung der Aufnahme über Nahrungsmittel - als Hauptaufnahmeweg der allgemeinen Bevölkerung - für
Deutschland eine deutliche Abnahme [3, $4,5,6,7]$, die auch in anderen Ländern beobachtet wurde [8]. Auch für PCB-Gehalte in der Muttermilch zeigt sich ein ähnlicher Zeitverlauf bei der Betrachtung der Ergebnisse der Untersuchungsprogramme in verschiedenen Bundesländern [9, $10,11,12,13]$. Insgesamt kann von einer deutlichen Minderung der PCB-Körperlast im Zeitraum nach Inkrafttreten der PCB-Beschränkungen am Ende der 70er Jahre ausgegangen werden. Die aktuell zu erwartende PCB-Körperlast wird (a) durch den Geburtszeitpunkt aufgrund der Unterschiede in der lebenslangen Expositionsbiographie, (b) bei gestillten Kindern durch die Expositionsbiographie der Mütter, (c) durch das Alter (Kumulation der PCB-Körperlast über die Lebenszeit) und (d) durch Zeiteffekte (tendenzielle Abnahme der inhalativen und oralen Exposition nach 1980 durch Abnahme der Umweltbelastung) beeinflusst. Eine zuverlässige quantitative Schätzung der zu erwartenden inneren PCB-Belastung der Bevölkerung ist allerdings ohne ausreichende empirische Daten nicht zu leisten.

Die HBM-Kommission stand nun vor der Situation, zur Beschreibung der heute $\mathrm{zu}$ erwartenden PCB-Belastung keine aktuellen bevölkerungsrepräsentativen Daten auswerten zu können, da entspre-

Tab. 1 Zeitlicher Trend der Plasmakonzentration von PCB 138 in Proben der Umweltprobenbank

\begin{tabular}{|c|c|c|c|c|c|c|c|c|}
\hline$\mu \mathrm{g} / \mathrm{l}$ Plasma & Frauen & $\mathrm{N}=3$ & & & Männer & $\mathrm{N}=$ & & \\
\hline Jahr & $\mathrm{N}$ & gM & gSD & 95. Perz. & $N$ & gM & gSD & 95. Perz. \\
\hline 1997 & 196 & 0,65 & 1,61 & 1,42 & 168 & 0,80 & 1,58 & 1,52 \\
\hline 1998 & 167 & 0,60 & 1,88 & 1,69 & 184 & 0,73 & 1,80 & 1,80 \\
\hline 1999 & 229 & 0,62 & 1,68 & 1,35 & 165 & 0,71 & 1,69 & 1,68 \\
\hline 2000 & 231 & 0,64 & 1,45 & 1,14 & 154 & 0,70 & 1,37 & 1,10 \\
\hline 2001 & 211 & 0,63 & 1,83 & 1,47 & 172 & 0,82 & 1,69 & 1,67 \\
\hline 2002 & 247 & 0,53 & 1,51 & 1,01 & 196 & 0,55 & 1,54 & 1,20 \\
\hline 2003 & 247 & 0,61 & 1,51 & 1,18 & 189 & 0,62 & 1,47 & 1,11 \\
\hline 2004 & 260 & 0,48 & 1,56 & 1,05 & 183 & 0,54 & 1,45 & 1,02 \\
\hline 2005 & 243 & 0,49 & 1,56 & 1,00 & 194 & 0,51 & 1,46 & 0,87 \\
\hline 2006 & 227 & 0,37 & 1,59 & 0,78 & 178 & 0,42 & 1,54 & 0,85 \\
\hline 2007 & 263 & 0,40 & 1,57 & 0,84 & 167 & 0,41 & 1,55 & 0,83 \\
\hline 2008 & 245 & 0,34 & 1,57 & 0,69 & 204 & 0,37 & 1,49 & 0,75 \\
\hline 2009 & 232 & 0,23 & 1,64 & 0,52 & 194 & 0,25 & 1,70 & 0,64 \\
\hline 2010 & 276 & 0,15 & 1,69 & 0,39 & 181 & 0,17 & 1,58 & 0,35 \\
\hline
\end{tabular}

N Anzahl untersuchter Proben, gM geom. Mittelwert, gSD geom. Standardabweichung, 95 . Perz. empirische Schätzung des Datenbereichs unter/gleich dem $95 \%$ der Verteilung liegt. 
Tab. 2 Zeitlicher Trend der Plasmakonzentration von PCB 153 in Proben der Umweltprobenbank

\begin{tabular}{|c|c|c|c|c|c|c|c|c|}
\hline$\mu \mathrm{g} / \mathrm{l}$ Plasma & Frauen & $\mathrm{N}=3$ & & & Männer & $\mathrm{N}=2$ & & \\
\hline Jahr & $\mathbf{N}$ & $g M$ & gSD & 95. Perz. & $\mathrm{N}$ & gM & gSD & 95. Perz. \\
\hline 1997 & 196 & 0,44 & 1,58 & 0,92 & 168 & 0,55 & 1,53 & 1,03 \\
\hline 1998 & 167 & 0,41 & 1,72 & 0,92 & 184 & 0,50 & 1,70 & 1,12 \\
\hline 1999 & 229 & 0,40 & 1,51 & 0,76 & 165 & 0,48 & 1,53 & 1,01 \\
\hline 2000 & 231 & 0,48 & 1,43 & 0,87 & 154 & 0,54 & 1,35 & 0,86 \\
\hline 2001 & 211 & 0,40 & 1,67 & 0,82 & 172 & 0,49 & 1,60 & 0,91 \\
\hline 2002 & 247 & 0,32 & 1,45 & 0,56 & 196 & 0,34 & 1,50 & 0,62 \\
\hline 2003 & 247 & 0,32 & 1,53 & 0,63 & 189 & 0,32 & 1,51 & 0,56 \\
\hline 2004 & 260 & 0,28 & 1,53 & 0,60 & 183 & 0,31 & 1,45 & 0,59 \\
\hline 2005 & 243 & 0,30 & 1,57 & 0,63 & 194 & 0,31 & 1,48 & 0,56 \\
\hline 2006 & 227 & 0,25 & 1,58 & 0,52 & 178 & 0,28 & 1,53 & 0,57 \\
\hline 2007 & 263 & 0,24 & 1,60 & 0,56 & 167 & 0,25 & 1,62 & 0,53 \\
\hline 2008 & 245 & 0,21 & 1,58 & 0,44 & 204 & 0,24 & 1,51 & 0,48 \\
\hline 2009 & 232 & 0,18 & 1,64 & 0,37 & 194 & 0,19 & 1,70 & 0,45 \\
\hline 2010 & 276 & 0,17 & 1,63 & 0,36 & 181 & 0,19 & 1,57 & 0,41 \\
\hline
\end{tabular}

N Anzahl untersuchter Proben, gM geom. Mittelwert, gSD geom. Standardabweichung, 95. Perz. empirische Schätzung des Datenbereichs unter/gleich dem $95 \%$ der Verteilung liegt.

Tab. 3 Zeitlicher Trend der Plasmakonzentration von PCB 180 in Proben der Umweltprobenbank

\begin{tabular}{|c|c|c|c|c|c|c|c|c|}
\hline$\mu \mathrm{g} / \mathrm{I}$ Plasma & Frauen & $\mathrm{N}=3$ & & & Männer & $\mathrm{N}=2$ & & \\
\hline Jahr & $\mathrm{N}$ & gM & gSD & 95. Perz. & $\mathrm{N}$ & $g M$ & gSD & 95. Perz. \\
\hline 1997 & 196 & 0,29 & 1,59 & 0,64 & 168 & 0,40 & 1,66 & 0,87 \\
\hline 1998 & 167 & 0,22 & 1,90 & 0,56 & 184 & 0,31 & 1,65 & 0,71 \\
\hline 1999 & 229 & 0,21 & 1,64 & 0,46 & 165 & 0,27 & 1,76 & 0,64 \\
\hline 2000 & 231 & 0,18 & 1,62 & 0,39 & 154 & 0,23 & 1,61 & 0,45 \\
\hline 2001 & 211 & 0,27 & 1,69 & 0,60 & 172 & 0,34 & 1,60 & 0,73 \\
\hline 2002 & 247 & 0,18 & 1,54 & 0,35 & 196 & 0,21 & 1,56 & 0,45 \\
\hline 2003 & 247 & 0,15 & 1,74 & 0,34 & 189 & 0,17 & 1,77 & 0,38 \\
\hline 2004 & 260 & 0,14 & 1,65 & 0,30 & 183 & 0,17 & 1,52 & 0,35 \\
\hline 2005 & 243 & 0,14 & 1,77 & 0,32 & 194 & 0,16 & 1,66 & 0,35 \\
\hline 2006 & 227 & 0,12 & 1,70 & 0,29 & 178 & 0,15 & 1,65 & 0,37 \\
\hline 2007 & 263 & 0,11 & 1,78 & 0,30 & 167 & 0,13 & 1,82 & 0,30 \\
\hline 2008 & 245 & 0,10 & 1,76 & 0,25 & 204 & 0,12 & 1,65 & 0,27 \\
\hline 2009 & 232 & 0,12 & 1,83 & 0,35 & 194 & 0,14 & 1,72 & 0,33 \\
\hline 2010 & 276 & 0,11 & 1,71 & 0,28 & 181 & 0,13 & 1,64 & 0,34 \\
\hline
\end{tabular}

N Anzahl untersuchter Proben, gM geom. Mittelwert, gSD geom. Standardabweichung, 95. Perz. empirische Schätzung des Datenbereichs unter/gleich dem $95 \%$ der Verteilung liegt.

chende Surveydaten nicht vorliegen. Um diesem Umstand Rechnung zu tragen, hat die HBM-Kommission die Bundesländer gebeten, die dort gemessenen Daten aus den vergangenen Jahren für eine Aktualisierung zur Verfügung zu stellen. Eine Datensichtung auf der Länderebene ergab, dass eine Neuberech- nung der $\mathrm{RV}_{95}$ für PCB nicht möglich ist, weil die vorliegende Datengrundlage vom Stichprobenumfang und von der -zusammensetzung nicht als ausreichend bevölkerungsrepräsentativ einzustufen ist und z. T. nur anlassbezogen ausgewählte Bevölkerungsgruppen untersucht wurden. Wegen der geringen
Fallzahlen wäre zudem eine Stratifizierung nach Lebensalter und Geschlecht kaum möglich $[14,15]$. Auch die Erhebungsmethoden der Labordaten waren nicht einheitlich (Vollblutwerte, Plasmawerte oder Werte bezogen auf den Blutfettgehalt). Die Sichtung der vorliegenden Daten aus den Bundesländern weist 
Tab. 4 Zeitlicher Trend der Plasmakonzentration von (PCB 138 + PCB $153+$ PCB 180) $\times 2$ in Proben der Umweltprobenbank

\begin{tabular}{llllllllll}
\hline $\boldsymbol{\mu g} /$ I Plasma & Frauen & $\mathbf{N}=\mathbf{3 2 7 4}$ & & \multicolumn{3}{l}{ Männer } & $\mathbf{N}=\mathbf{2 5 2 9}$ \\
\hline Jahr & $\mathbf{N}$ & $\mathbf{g M}$ & $\mathbf{g S D}$ & $\mathbf{9 5 .}$ Perz. & $\mathbf{N}$ & $\mathbf{g M}$ & $\mathbf{g S D}$ & $\mathbf{9 5 .}$ Perz. \\
\hline 1997 & 196 & 2,79 & 1,56 & 6,12 & 168 & 3,54 & 1,53 & 7,00 \\
\hline 1998 & 167 & 2,52 & 1,73 & 6,10 & 184 & 3,14 & 1,67 & 6,93 \\
\hline 1999 & 229 & 2,49 & 1,57 & 4,83 & 165 & 2,94 & 1,59 & 6,40 \\
\hline 2000 & 231 & 2,61 & 1,44 & 4,89 & 154 & 2,96 & 1,38 & 4,68 \\
\hline 2001 & 211 & 2,66 & 1,68 & 5,40 & 172 & 3,35 & 1,59 & 6,36 \\
\hline 2002 & 247 & 2,08 & 1,46 & 3,65 & 196 & 2,23 & 1,49 & 4,38 \\
\hline 2003 & 247 & 2,17 & 1,53 & 4,32 & 189 & 2,25 & 1,51 & 4,00 \\
\hline 2004 & 260 & 1,79 & 1,55 & 3,81 & 183 & 2,06 & 1,45 & 4,08 \\
\hline 2005 & 243 & 1,86 & 1,58 & 3,83 & 194 & 1,96 & 1,49 & 3,59 \\
\hline 2006 & 227 & 1,48 & 1,59 & 3,20 & 178 & 1,72 & 1,54 & 3,46 \\
\hline 2007 & 263 & 1,52 & 1,58 & 3,33 & 167 & 1,60 & 1,58 & 3,29 \\
\hline 2008 & 245 & 1,32 & 1,58 & 2,83 & 204 & 1,47 & 1,51 & 3,00 \\
\hline 2009 & 232 & 1,08 & 1,61 & 2,49 & 194 & 1,19 & 1,64 & 2,77 \\
\hline 2010 & 276 & 0,87 & 1,60 & 1,98 & 181 & 0,99 & 1,55 & 2,08 \\
\hline
\end{tabular}

N Anzahl untersuchter Proben, $g$ M geom. Mittelwert, $g$ SD geom. Standardabweichung, 95 . Perz. empirische Schätzung des Datenbereichs unter/gleich dem $95 \%$ der Verteilung liegt.

Tab. 5 PCB-Plasmakonzentrationen einer Stichprobe nicht beruflich exponierter Probanden [23]

\begin{tabular}{|c|c|c|c|c|c|c|}
\hline$\mu \mathrm{g} / \mathrm{I}$ Plasma & PCB 138 & & PCB 153 & & PCB 180 & \\
\hline & Median & 95. Perzentil & Median & 95. Perzentil & Median & 95.Perzentil \\
\hline$\leq 10$ Jahre & 0,17 & 0,47 & 0,26 & 0,67 & 0,17 & 0,42 \\
\hline $11-20$ & 0,12 & 0,38 & 0,15 & 0,54 & 0,10 & 0,35 \\
\hline $21-30$ & 0,21 & 0,47 & 0,27 & 0,60 & 0,17 & 0,48 \\
\hline $31-40$ & 0,36 & 0,78 & 0,53 & 1,04 & 0,42 & 0,70 \\
\hline $41-49$ & 0,35 & 1,24 & 0,53 & 1,67 & 0,53 & 1,61 \\
\hline $51-60$ & 0,61 & 1,67 & 0,90 & 2,01 & 0,89 & 1,68 \\
\hline$>60$ Jahre & 0,87 & 1,54 & 1,34 & 2,22 & 1,29 & 1,88 \\
\hline
\end{tabular}

gleichwohl darauf hin, dass gegenüber den $\mathrm{RV}_{95}$ von 1997/99 [2] eine deutliche Abnahme der Grundbelastung der Bevölkerung mit PCB wahrscheinlich ist. Die genaue Größenordnung kann jedoch an Hand der vorliegenden $\mathrm{Hu}-$ man-Biomonitoring-Daten aus den Bundesländern alleine nicht verlässlich eingeschätzt werden.

Die HBM-Kommission hat daraufhin geprüft, ob die Daten der Umweltprobenbank (UPB) aus den Jahren 1997 bis $2010[16,17]$ geeignet sind, die $\mathrm{RV}_{95}$ von 1997/99 [2] durch Berechnung eines Minderungsfaktors auf den heutigen Stand zu übertragen. Die Daten der UPB sind im Jahresbericht [18] und im Internet unter http://www.umweltpro- benbank.de/de verfügbar. Der von der HBM-Kommission analysierte Datensatz umfasst $\mathrm{N}=5803$ auswertbare PCB-Messungen bei Studenten $(\mathrm{N}=2529)$ und Studentinnen $(\mathrm{N}=3274)$ im Alter von 20-29 Jahren aus den Universitätsstädten Münster/Westfalen, Halle/Saale, Greifswald und Ulm (1997-2010). Die hier dargestellten 0 Tabellen 1-4 beschreiben den zeitlichen Trend der Plasmakonzentrationen. In der Publikation der Umweltprobenbank wird darauf hingewiesen, dass die Plasmakonzentrationen von Probanden aus den Universitätsstädten Münster und Ulm über denen der Probanden aus Halle/Saale und Greifswald lagen. Es bestehen signifikante Niveau-Unterschiede zwischen neuen und alten Bundesländern in Bezug auf die PCB-Belastung. Die Autoren gehen von einem stärkeren Einfluss des Geburtsortes als des Wohnortes aus. In den von der HBM-Kommission durchgeführten Regressionsanalysen wurden für alle betrachteten PCB signifikant höhere Konzentrationen bei männlichen Studienteilnehmern gesehen, wobei sich dieser Unterschied absolut und relativ über die Jahre vermindert hat. Über das Alter steigt im multiplen Regressionsmodell bei den PCB-Kongeneren die Plasmakonzentration hoch signifikant an, die Effektstärke ist gegenüber dem Einfluss der Untersuchungsjahre bzw. der jeweiligen Geburtsjahre der Studenten/innen relativ gering. 
Tab. 6 Anlassbezogen gemessene PCB-Konzentrationen im Blutplasma und im Blutfett, Südbayern [24]

\begin{tabular}{|lllllllll}
\hline & PCB 138 & \multicolumn{3}{c}{ PCB 153 } & PCB 180 & \multicolumn{3}{c}{ Summe PCB $^{\text {a }}$} \\
\hline & Median & 95.Perz. & Median & 95.Perz. & Median & 95.Perz. & Median & 95.Perz. \\
\hline$\mu \mathrm{g} / \mathrm{l} \mathrm{Plasma}$ & 0,08 & 0,55 & 0,15 & 1,00 & 0,15 & 1,02 & 0,77 & 4,90 \\
\hline$\mu \mathrm{g} \mathrm{g}^{-1}$ Fett & 0,02 & 0,10 & 0,03 & 0,17 & 0,03 & 0,18 & 0,17 & 0,84 \\
\hline
\end{tabular}

a: Summe von PCB 138, PCB 153 und PCB 180, multipliziert mit dem Faktor 2

Tab. 7 PCB 138 im Plasma der Allgemeinbevölkerung [25]

\begin{tabular}{|c|c|c|c|c|c|c|c|c|}
\hline $\begin{array}{l}\mu \mathrm{g} / \mathrm{l} \\
\text { Plasma }\end{array}$ & Frauen & $\mathrm{N}=1$ & & & Männer & $N=1$ & & \\
\hline Alter & $\mathrm{N}$ & gM & gSD & 95. Perz. & $\mathbf{N}$ & gM & gSD & 95. Perz. \\
\hline$<10$ & 33 & 0,05 & 1,93 & 0,14 & 34 & 0,05 & 1,80 & 0,18 \\
\hline $10-19$ & 316 & 0,09 & 1,71 & 0,21 & 250 & 0,10 & 1,78 & 0,24 \\
\hline $20-29$ & 275 & 0,13 & 1,65 & 0,29 & 218 & 0,13 & 1,64 & 0,27 \\
\hline $30-39$ & 228 & 0,18 & 1,62 & 0,37 & 252 & 0,17 & 1,74 & 0,38 \\
\hline $40-49$ & 245 & 0,28 & 1,67 & 0,58 & 221 & 0,31 & 1,66 & 0,76 \\
\hline $50-59$ & 304 & 0,44 & 1,68 & 1,07 & 216 & 0,46 & 1,73 & 1,08 \\
\hline $60+$ & 71 & 0,67 & 1,79 & 1,32 & 100 & 0,62 & 1,72 & 1,36 \\
\hline
\end{tabular}

N Anzahl untersuchter Proben, gM geom. Mittelwert, gSD geom. Standardabweichung, 95. Perz. empirische Schätzung des Datenbereichs unter/gleich dem $95 \%$ der Verteilung liegt.

Tab. 8 PCB 153 im Plasma der Allgemeinbevölkerung [25]

\begin{tabular}{|c|c|c|c|c|c|c|c|c|}
\hline$\mu \mathrm{g} / \mathrm{l}$ Plasma & Frauen & $\mathrm{N}=1$ & & & Männer & $\mathrm{N}=1$ & & \\
\hline Alter & $\mathrm{N}$ & gM & gSD & 95. Perz. & $\mathrm{N}$ & gM & gSD & 95. Perz. \\
\hline$<10$ & 33 & 0,06 & 2,00 & 0,19 & 34 & 0,07 & 1,95 & 0,29 \\
\hline $10-19$ & 316 & 0,12 & 1,84 & 0,31 & 250 & 0,13 & 1,93 & 0,37 \\
\hline $20-29$ & 275 & 0,19 & 1,71 & 0,43 & 218 & 0,18 & 1,68 & 0,40 \\
\hline $30-39$ & 228 & 0,25 & 1,65 & 0,56 & 252 & 0,25 & 1,75 & 0,56 \\
\hline $40-49$ & 245 & 0,43 & 1,73 & 0,90 & 221 & 0,49 & 1,66 & 1,21 \\
\hline $50-59$ & 304 & 0,70 & 1,68 & 1,58 & 216 & 0,76 & 1,71 & 1,76 \\
\hline $60+$ & 71 & 1,07 & 1,74 & 2,06 & 100 & 1,03 & 1,66 & 2,53 \\
\hline
\end{tabular}

N Anzahl untersuchter Proben, gM geom. Mittelwert, gSD geom. Standardabweichung, 95. Perz. empirische Schätzung des Datenbereichs unter/gleich dem $95 \%$ der Verteilung liegt.

Die HBM-Kommission kommt $\mathrm{zu}$ dem Schluss, dass dieser Datensatz [17, 18] geeignet ist, zumindest für den Altersbereich der 20- bis 29-Jährigen die Veränderung der PCB-Gehalte im Blut über die Zeit, die Einflüsse der Geburtsjahrgänge (Expositionsbiografie) und des Lebensalters zusammen mit Labordaten zu Blutfetten zu schätzen. Berichtet wird hier geschlechtsdifferenziert der zeitliche Trend der geometrischen Mittelwerte, der geometrischen Standabweichungen und der $95 \%$-Perzentile. Für den Altersbereich der 20- bis 29-jährigen Studierenden sieht die HBM-Kommission für den Zeitraum 1997 - 2010 eine Minderung der
PCB-Plasmakonzentrationen (Kongenere $138,153,180)$ auf etwa ein Drittel (Faktor 0,33$)$.

Dieser Faktor gilt für die PCB-Gehalte im Blut der Altersgruppe etwa bis zum 30. Lebensjahr. Für ältere Personen mit einer langen Expositionsbiografie geht die HBM-Kommission von einer geringeren Minderung mit einem Faktor von etwa 0,5 aus. Die Annahme eines geringeren Abfalls für die PCB-Gehalte im Blut der älteren Altersgruppen etwa ab dem 30. Lebensjahr gründet auf der Tatsache, dass die Plasma-Halbwertszeiten für die betrachteten Kongenere etwa bei 10 Jahren liegen $[19,20]$ und die längere
Expositionsbiografie dieser Altersgruppen, insbesondere die höhere Aufnahmerate in den Jahren mit hoher Kontamination, eine höhere Körperlast bedingt [21, 22].

In aktuelleren Arbeiten wurden PCB-Konzentrationen für ausgewählte Stichproben aus Deutschland berichtet. Schettgen et al. [23] zeigen für eine Stichprobe nicht beruflich exponierter Probanden für den Zeitraum 2003-2004 (N=105 Datensätze, Pilotstudie) die der

- Tabelle 5 zu entnehmenden Ergebnisse.

Fromme et al. [24] berichten auf der Datengrundlage einer anlassbezogenen Untersuchung aus Südbayern (70 Teilneh- 
Tab. 9 PCB 180 im Plasma der Allgemeinbevölkerung [25]

\begin{tabular}{|c|c|c|c|c|c|c|c|c|}
\hline$\mu \mathrm{g} / \mathrm{l}$ Plasma & Frauen & $\mathbf{N}=1$ & & & Männer & $\mathrm{N}=1$ & & \\
\hline Alter & $\mathrm{N}$ & $g M$ & gSD & 95. Perz. & $\mathrm{N}$ & $\mathrm{gM}$ & gSD & 95. Perz. \\
\hline$<10$ & 33 & 0,03 & 2,37 & 0,10 & 34 & 0,03 & 2,21 & 0,17 \\
\hline $10-19$ & 316 & 0,06 & 2,12 & 0,22 & 250 & 0,08 & 2,25 & 0,26 \\
\hline $20-29$ & 275 & 0,11 & 1,85 & 0,31 & 218 & 0,12 & 1,84 & 0,33 \\
\hline $30-39$ & 228 & 0,17 & 1,72 & 0,37 & 252 & 0,17 & 1,85 & 0,43 \\
\hline $40-49$ & 245 & 0,34 & 1,78 & 0,80 & 221 & 0,43 & 1,62 & 0,93 \\
\hline $50-59$ & 304 & 0,63 & 1,65 & 1,38 & 216 & 0,75 & 1,62 & 1,63 \\
\hline $60+$ & 71 & 0,94 & 1,77 & 1,91 & 100 & 1,01 & 1,63 & 2,27 \\
\hline
\end{tabular}

N Anzahl untersuchter Proben, gM geom. Mittelwert, gSD geom. Standardabweichung, 95. Perz. empirische Schätzung des Datenbereichs unter/gleich dem $95 \%$ der Verteilung liegt.

Tab. 10 (PCB $138+$ PCB $153+$ PCB 180) ×2 im Plasma der Allgemeinbevölkerung [25]

\begin{tabular}{|c|c|c|c|c|c|c|c|c|}
\hline$\mu \mathrm{g} / \mathrm{l}$ Plasma & Frauen & $\mathrm{N}=$ & & & & Männer & $\mathrm{N}=1$ & \\
\hline Alter & $\mathrm{N}$ & gM & gSD & 95.Perz. & $N$ & gM & gSD & 95.Perz. \\
\hline$<10$ & 33 & 0,29 & 2,03 & 0,86 & 34 & 0,31 & 1,96 & 1,33 \\
\hline $10-19$ & 316 & 0,54 & 1,85 & 1,48 & 250 & 0,62 & 1,94 & 1,67 \\
\hline $20-29$ & 275 & 0,87 & 1,71 & 2,06 & 218 & 0,86 & 1,69 & 1,98 \\
\hline 30-39 & 228 & 1,19 & 1,64 & 2,62 & 252 & 1,18 & 1,75 & 2,63 \\
\hline $40-49$ & 245 & 2,12 & 1,70 & 4,60 & 221 & 2,49 & 1,62 & 5,84 \\
\hline 50-59 & 304 & 3,57 & 1,63 & 8,09 & 216 & 3,98 & 1,64 & 8,54 \\
\hline $60+$ & 71 & 5,42 & 1,72 & 10,50 & 100 & 5,40 & 1,61 & 12,10 \\
\hline
\end{tabular}

N Anzahl untersuchter Proben, gM geom. Mittelwert, $g$ SD geom. Standardabweichung, 95. Perz. empirische Schätzung des Datenbereichs unter/gleich dem $95 \%$ der Verteilung liegt.

mer/innen: 37 Frauen und 33 Männer, Altersbereich 4-76 Jahre) für das Jahr 2013 die in - Tabelle 6 dargestellten Mediane und $95 \%$-Perzentile.

Eine deskriptive Darstellung des im Rahmen einer Untersuchungsreihe $\mathrm{zu}$ niederchlorierten PCB im Innenraum erhobenen Datensatzes zu PCB-Plasmakonzentrationen aus den Jahren 2010-2014 findet sich bei Schettgen et al. [25]. Auch wenn dieser Datenbestand aus Selbstanmeldungen von Personen stammt, die eine PCB-Exposition als möglich ansahen, kann aus der Verteilung der Konzentrationen eine mögliche Abnahme der Mediane und, vorsichtig interpretiert, der $95 \%$-Perzentile gefolgert werden. Für eine Referenzwert-Ableitung ist der Datenbestand jedoch nicht geeignet. Um mögliche Unterschiede über das Geschlecht (entsprechend den bisherigen Analysen) berücksichtigen zu können, wurde der von den Autoren zur Verfügung gestellte Datensatz durch die HBM-Kommission für
PCB 138, PCB 153 und PCB 180 reanalysiert.

Für die PCB 138-Plasmakonzentrationen zeigte sich kein statistisch bedeutsamer Unterschied zwischen den männlichen und weiblichen Teilnehmer/innen. Die $95 \%$-Perzentile liegen im Datensatz von Schettgen et al. [25] geringfügig unter den jetzt vorgeschlagenen Referenzwerten (- Tabellen 7 und 11).

Für die PCB 153-Plasmakonzentrationen zeigte sich ein statistisch nicht bedeutsamer Trend zu höheren Plasmakonzentrationen bei den männlichen in Relation zu den weiblichen Teilnehmer/-innen $(p=0,08)$. Die $95 \%$-Perzentile liegen im Datensatz von Schettgen et al. [25] geringfügig unter den jetzt vorgeschlagenen Referenzwerten ( $\bullet$ Tabellen 8 und 11).

Die Verteilung der PCB 180-Plasmakonzentrationen (logarithmierte Daten) weist für die männlichen Studienteilnehmer auf eine signifikant höhere PCB-Plasmakonzentration hin $(p<0,001)$. Die geometrischen Mittelwer- te und die $95 \%$-Perzentile liegen für Männer höher. In Relation zu den jetzt von der HBM-Kommission vorgelegten Referenzwerten (• Tabelle 11) liegen die 95\%-Perzentile aus dem Datensatz von Schettgen et al. (- Tabelle 9, [25]) etwa in der gleichen Größenordnung.

Für die Summe der PCB $(138+153+180) \times$ Faktor 2 (entsprechend der Angabe der HBM-Werte [26]) zeigen sich im Datensatz von Schettgen et al. (• Tabelle 10, [25]) in den jüngeren Altersgruppen ebenfalls signifikante Unterschiede zwischen den Geschlechtsgruppen $(\mathrm{p}=0,007)$ mit höheren PCB-Plasmakonzentrationen in der Gruppe der männlichen Teilnehmer.

Da seit der Probenahme für die zuletzt aktualisierten Referenzwerte für Erwachsene [2] mehr als 15 Jahre vergangen sind, wäre bei einer Plasma-Halbwertszeit von 10 Jahren eine Minderung der 1997 gemessenen Konzentrationen auf etwa $34 \%$ zu erwarten. Da die Expositionsrate sich über die Zeit deutlich gesenkt hat, scheint 
Tab. 11 Referenzwerte (RV95) für polychlorierte Biphenyle (PCB) im Blut

\begin{tabular}{|c|c|c|c|c|c|c|c|c|c|}
\hline \multicolumn{10}{|c|}{ Aktualisierung } \\
\hline Parameter & $\begin{array}{l}\text { Population/ } \\
\text { Altersgruppe }\end{array}$ & $\begin{array}{l}\text { Jahr der } \\
\text { Studie }\end{array}$ & $\begin{array}{l}\text { RV95 } \\
\text { in } \mu g / l \\
\text { Vollblut }\end{array}$ & $\begin{array}{l}\text { Jahr der } \\
\text { Studie }\end{array}$ & $\begin{array}{l}\text { Männer/ } \\
\text { Frauen } \\
\mu \mathrm{g} / \mathrm{l} \\
\text { Vollblut }\end{array}$ & $\begin{array}{l}\text { Männer } \\
\mu \mathrm{g} / \mathrm{l} \\
\text { Plasma }\end{array}$ & $\begin{array}{l}\text { Frauen } \\
\mu \mathrm{g} / \mathrm{l} \\
\text { Plasma }\end{array}$ & $\begin{array}{l}\text { Männer } \\
\mu \mathrm{g} / \mathrm{g} \\
\text { Fett }\end{array}$ & $\begin{array}{l}\text { Frauen } \\
\mu \mathrm{g} / \mathrm{g} \\
\text { Fett }\end{array}$ \\
\hline PCB 138 & 7-14 Jahre ${ }^{1}$ & $2003 / 06$ & 0,3 & & & & & & \\
\hline \multirow[t]{6}{*}{ [1999, 2001, 2009] } & 18-19 Jahre ${ }^{2}$ & $1997 / 99$ & 0,4 & $2010^{3}$ & 0,13 & 0,24 & 0,22 & 0,035 & 0,032 \\
\hline & 20-29 Jahre ${ }^{2}$ & 1997/99 & 0,6 & $2010^{3}$ & 0,20 & 0,36 & 0,33 & 0,052 & 0,048 \\
\hline & 30-39 Jahre ${ }^{2}$ & 1997/99 & 0,9 & $2010^{3}$ & 0,45 & 0,82 & 0,75 & 0,12 & 0,11 \\
\hline & 40-49 Jahre ${ }^{2}$ & $1997 / 99$ & 1,4 & $2010^{3}$ & 0,70 & 1,3 & 1,2 & 0,18 & 0,17 \\
\hline & 50-59 Jahre ${ }^{2}$ & $1997 / 99$ & 1,7 & $2010^{3}$ & 0,85 & 1,5 & 1,4 & 0,22 & 0,20 \\
\hline & 60-69 Jahre ${ }^{2}$ & $1997 / 99$ & 2,2 & $2010^{3}$ & 1,10 & 2,0 & 1,8 & 0,29 & 0,26 \\
\hline РCB 153 & 7-14 Jahre ${ }^{1}$ & $2003 / 06$ & 0,4 & & & & & & \\
\hline \multirow[t]{6}{*}[1999,2001,2009]{} & 18-19 Jahre ${ }^{2}$ & $1997 / 99$ & 0,6 & $2010^{3}$ & 0,20 & 0,36 & 0,33 & 0,052 & 0,048 \\
\hline & 20-29 Jahre ${ }^{2}$ & 1997/99 & 0,9 & $2010^{3}$ & 0,30 & 0,54 & 0,50 & 0,078 & 0,071 \\
\hline & 30-39 Jahre ${ }^{2}$ & $1997 / 99$ & 1,6 & $2010^{3}$ & 0,80 & 1,5 & 1,3 & 0,21 & 0,19 \\
\hline & 40-49 Jahre ${ }^{2}$ & $1997 / 99$ & 2,2 & $2010^{3}$ & 1,10 & 2,0 & 1,8 & 0,29 & 0,26 \\
\hline & 50-59 Jahre ${ }^{2}$ & $1997 / 99$ & 2,8 & $2010^{3}$ & 1,40 & 2,5 & 2,3 & 0,36 & 0,33 \\
\hline & $60-69$ Jahre $^{2}$ & 1997/99 & 3,3 & $2010^{3}$ & 1,65 & 3,0 & 2,8 & 0,43 & 0,39 \\
\hline PCB 180 & 7-14 Jahre ${ }^{1}$ & $2003 / 06$ & 0,3 & & & & & & \\
\hline \multirow[t]{6}{*}[1999,2001,2009]{} & 18-19 Jahre ${ }^{2}$ & 1997/99 & 0,3 & $2010^{3}$ & 0,10 & 0,18 & 0,17 & 0,026 & 0,024 \\
\hline & 20-29 Jahre ${ }^{2}$ & $1997 / 99$ & 0,6 & $2010^{3}$ & 0,20 & 0,36 & 0,33 & 0,052 & 0,048 \\
\hline & 30-39 Jahre ${ }^{2}$ & 1997/99 & 1 & $2010^{3}$ & 0,50 & 0,91 & 0,83 & 0,13 & 0,12 \\
\hline & 40-49 Jahre ${ }^{2}$ & 1997/99 & 1,6 & $2010^{3}$ & 0,80 & 1,5 & 1,3 & 0,21 & 0,19 \\
\hline & 50-59 Jahre ${ }^{2}$ & 1997/99 & 2,1 & $2010^{3}$ & 1,05 & 1,9 & 1,8 & 0,27 & 0,25 \\
\hline & 60-69 Jahre ${ }^{2}$ & 1997/99 & 2,4 & $2010^{3}$ & 1,2 & 2,2 & 2,0 & 0,31 & 0,29 \\
\hline$\Sigma \mathrm{PCB}$ & 7-14 Jahre ${ }^{1}$ & $2003 / 06$ & 1 & & & & & & \\
\hline$(138+153+180)$ & 18-19 Jahre ${ }^{2}$ & $1997 / 99$ & 1,1 & $2010^{3}$ & 0,37 & 0,67 & 0,61 & 0,10 & 0,087 \\
\hline \multirow{5}{*}{$\begin{array}{l}{[1999,2001,2009,} \\
2012]\end{array}$} & 20-29 Jahre ${ }^{2}$ & 1997/99 & 2 & $2010^{3}$ & 0,67 & 1,2 & 1,1 & 0,17 & 0,16 \\
\hline & 30-39 Jahre ${ }^{2}$ & $1997 / 99$ & 3,2 & $2010^{3}$ & 1,6 & 2,9 & 2,7 & 0,42 & 0,38 \\
\hline & 40-49 Jahre ${ }^{2}$ & $1997 / 99$ & 5,1 & $2010^{3}$ & 2,6 & 4,6 & 4,3 & 0,66 & 0,61 \\
\hline & 50-59 Jahre ${ }^{2}$ & 1997/99 & 6,4 & $2010^{3}$ & 3,2 & 5,8 & 5,3 & 0,83 & 0,76 \\
\hline & 60-69 Jahre ${ }^{2}$ & 1997/99 & 7,8 & $2010^{3}$ & 3,9 & 7,1 & 6,5 & 1,0 & 0,93 \\
\hline$\Sigma \mathrm{PCB}$ & 7-14 Jahre ${ }^{1}$ & $2003 / 06$ & 2 & & & & & & \\
\hline \multirow{6}{*}{$\begin{array}{l}(138+153+180) x \\
\text { Faktor } 2[2012]\end{array}$} & 18-19 Jahre ${ }^{2}$ & 1997/99 & 2,2 & $2010^{3}$ & 0,73 & 1,3 & 1,2 & 0,19 & 0,17 \\
\hline & 20-29 Jahre ${ }^{3}$ & 2010 & 4 & $2010^{3}$ & 1,3 & 2,4 & 2,2 & 0,35 & 0,32 \\
\hline & 30-39 Jahre $^{3}$ & 2010 & 6,4 & $2010^{3}$ & 3,2 & 5,8 & 5,3 & 0,83 & 0,76 \\
\hline & 40-49 Jahre ${ }^{2}$ & 1997/99 & 10,2 & $2010^{3}$ & 5,1 & 9,3 & 8,5 & 1,3 & 1,2 \\
\hline & 50-59 Jahre ${ }^{2}$ & $1997 / 99$ & 12,8 & $2010^{3}$ & 6,4 & 12 & 11 & 1,7 & 1,5 \\
\hline & 60-69 Jahre ${ }^{2}$ & 1997/99 & 16,6 & $2010^{3}$ & 8,3 & 15 & 14 & 2,2 & 2,0 \\
\hline
\end{tabular}

Anmerkungen: Jahresangabe siehe Publikationen: http://www.uba.de/gesundheit/publikationen/index.htm\#khb

a bei der Anwendung von $\mathrm{RV}_{95}$ ist grundsätzlich die analytische Messunsicherheit zu berücksichtigen, d. h. bei der Bewertung von HBM-Messwerten ist sicherzustellen, dass die Analysen unter den Bedingungen der internen und externen Qualitätssicherung durchgeführt wurden;

'Datenquelle: Kinder-Umwelt-Survey 2003/06; ${ }^{2}$ Datenquelle: Umwelt-Survey 1997/99; ${ }^{3}$ Datenbasis: Umweltprobenbank

Letzte Aktualisierung: 12/2012 
eine Senkung der 1997 gemessenen Referenzwerte um den Faktor 0,5 vertretbar.

Die HBM-Kommission sieht die Unsicherheiten, die sich aus der Übertragung der bei Studierenden der Altersgruppe bis 30 Jahre gewonnenen Daten auf die Allgemeinbevölkerung ergeben. Sie sieht die eingeschränkte Repräsentativität der zusätzlich betrachteten Daten und ebenso die Schwächen der Anwendung von Modellrechnungen. Allerdings rechtfertigt die Sichtung der aktuellen Datenlage [27] zusammen mit zeitlichen Analysen aus Querschnittsstudien [21, 22, 23, 24, 25, $28,29]$ eine Reduzierung der Referenzwerte um ca. 50 \% für Erwachsene gegenüber dem 1998 publizierten Stand.

Um eine Bewertung der PCB-Gehalte im Plasma vornehmen zu können, wurden die Vollblut bezogenen $\mathrm{RV}_{95}$ von 1997/99 [2] unter Berücksichtigung der geschlechtsspezifischen Hämatokritwerte (0,45 für Männer und 0,4 für Frauen) umgerechnet. Um eine Bewertung der auf Gramm (g) Fett bezogenen PCB-Gehalte zu ermöglichen, wurde bei der Umrechnung berücksichtigt, dass näherungsweise von einem Gesamtfettgehalt von 7 g pro Liter Blut auszugehen wäre [26].

\section{Zusammenfassung}

Die HBM-Kommission ist sich bewusst, dass mit der Ableitung der neuen PCB-Referenzwerte über eine faktorielle Schätzung Unsicherheiten verbunden sind. Dies bezieht sich auf die Methodik, aber insbesondere auch auf die eingeschränkte Repräsentativität der zum Vergleich herangezogenen Daten. Eine Aktualisierung auf der Basis bevölkerungsrepräsentativer Daten wird daher als erforderlich angesehen. Eine Einordnung aktuell erhobener Daten auf der Grundlage von Referenzwerten der Jahre 1997/1999 [2] wäre gleichwohl fachlich kaum noch $\mathrm{zu}$ vertreten.

In der - Tabelle 11 sind die Ausgangs- $\mathrm{RV}_{95}$ von 1997/99 [2] zusammen mit den umgerechneten $\mathrm{RV}_{95}$-Werten für die PCB-Konzentrationen im Vollblut, im Plasma sowie bezogen auf den Blut-Fettgehalt dargestellt. Darüber hinaus sind $\mathrm{RV}_{95}$ für die Summe der PCB $(138+153+180)$ $\times$ Faktor 2 entsprechend der Angabe der HBM-Werte angegeben [26]. Die neuen
Referenzwerte bilden den in verschiedenen Datengrundlagen gesehenen Trend einer Minderung der PCB-Exposition ab und weichen nicht systematisch von vorliegenden Daten ab, sie sind jedoch aus den genannten Gründen als vorläufig anzusehen.

Die in der Tabelle aufgeführten $\mathrm{RV}_{95}$ für Kinder im Alter von 7-14 Jahren wurden basierend auf Erhebungen des Kinder-Umweltsurveys aus den Jahren 2003-2006 ermittelt und werden von der HBM-Kommission als ausreichend aktuell angesehen.

Danksagung. Die HBM-Kommission dankt Dr. R. Eckard, Prof. Dr. T. Göen und Dr. M. Schümann für die Erstellung des Textentwurfes, Prof. Dr. J. Angerer, Prof. Dr. H. Fromme, Dr. J. Hurraß, Dr. M. Kraft und Prof. Dr. M. Wilhelm für die ergänzenden Beiträge sowie Angela Lehmann und Petra Apel für die redaktionelle Bearbeitung. Die HBM-Kommission dankt darüber hinaus Prof. Dr. T. Schettgen für die Bereitstellung des Datenmaterials zur Reanalyse.

\section{Korrespondenzadresse:}

\section{Petra Apel}

Umweltbundesamt,

Geschäftsstelle HBM-Kommission

Corrensplatz 1

14195 Berlin

Email: petra.apel@uba.de

Tel.: 030-8903-1344

\section{Literaturverzeichnis}

1. Kommission Human-Biomonitoring des Umweltbundesamtes (1998) Referenzwerte für die PCB-Kongenere Nr. 138, 153, 180 und deren Summe im Humanblut. Bundesgesundheitsblatt Gesundheitsforschung Gesundheitsschutz 41(9):416. doi:10.1007/BF03044173

2. Kommission Human-Biomonitoring des Umweltbundesamtes (2003) Aktualisierung der Referenzwerte für PCB-138, -153, -180 im Vollblut sowie Referenzwerte für HCB, $\beta-\mathrm{HCH}$ und DDE im Vollblut. Bundesgesundheitsblatt Gesundheitsforschung Gesundheitsschutz 46(2):161-168

3. Kibler R, Lepschy-v. Gleissenthall J (1990) Zufuhr von Polychlorierten Biphenylen (PCB) über den Gesamtverzehr. Z Lebensm Unters Forsch 191:2134-2216

4. Wilhelm M, Schrey P, Wittsiepe J, Heinzow B (2002) Dietary intake of persistent organic pollutants (POPs) by German children using duplicate portion sampling. Int J Hyg Environ Health 204:359-362

5. Schäfer M, Petzold G, Ostendorp G, Schade G, Mohr S, Heinzow B (2000) Duplikatstudie und Humanbiomonitoring zur Feststellung der PCB-Belastung bei jungen Frauen. Umweltmed Forsch Prax 5:154-160

6. Heinzow B (2006) Schadstoffbelastung aus der Nahrung: Studie zur Gesamtaufnahme von po- lychlorierten Biphenylen (PCB) bei jungen Frauen. Landesamt für Gesundheit und Arbeitssicherheit des Landes Schleswig-Holstein, Kiel

7. Fromme H, Shahin N, Boehmer S, Albrecht M, Parlar H, Liebl B, Mayer R, Bolte G (2009) Nahrungsbedingte Zufuhr von nicht-dioxinähnlichen polychlorierten Biphenylen (PCB) in Bayern. Ergebnisse von INES (Integrated Exposure Assessment Survey). Gesundheitswesen 71(5):275-280

8. Bakker M, Baars AJ, Baumann B, Boon PE, Hoogerbrugge $R$ (2003) Indicator PCBs in foodstuff: occurence and dietary intake in the Netherlands at the end of the 20th century. RIVM (National Institute for Public Health and the Environment) report 639102025/2003 \& RIKILT (Institute of Food Safety), report 2003.014, Bilthofen/Wageningen

9. Fürst $P$ (2006) Dioxins, polychlorinated biphenyls and other organohalogen compounds in human milk. Levels, correlations, trends and exposure through breastfeeding. Mol Nutr Food Res 50:922-933

10. Raab U, Albrecht M, Preiss U, Völkel W, Schwegler U, Fromme H (2013) Organochlorine compounds, nitro musks and perfluorinated substances in breast milk - Results from Bavarian Monitoring of Breast Milk 2007/8. Chemosphere 93:461-467

11. Raab U, Preiss U, Albrecht M, Shahin N, Parlar H, Fromme H (2008) Concentrations of polybrominated diphenyl ethers, organochlorine compounds and nitro musks in mother's milk from Germany (Bavaria). Chemosphere 72:87-94

12. Niedersächsisches Landesgesundheitsamt (2014) Das Muttermilchuntersuchungsprogramm des NLGA von 1999 bis 2012: Ergebnisse zu Organochlorverbindungen und PBDE in der Muttermilch, Hannover. http://www.nlga.niedersachsen.de/ portal/live.php?navigation_id=27085\&article_id=19364\&_psmand $=20$. Zugegriffen: 22 . Juni 2016

13. Ministerium für Soziales, Gesundheit, Familie und Gleichstellung des Landes Schleswig-Holstein (MSGFG) (2013) 25 Jahre Muttermilch-Untersuchungsprogramm Schleswig-Holstein (ISSN 0935-4379)

14. Kommission Human-Biomonitoring des Umweltbundesamtes (1996) Konzept der Referenz- und Human-Biomonitoring-Werte (HBM) in der Umweltmedizin. Bundesgesundheitsblatt Gesundheitsforschung Gesundheitsschutz 39(6):221-224

15. Kommission Human-Biomonitoring des Umweltbundesamtes (2009) Addendum zum Konzept der Referenz- und Human-Biomonitoring-Werte in der Umweltmedizin. Stellungnahme der Kommission Human-Biomonitoring des Umweltbundesamtes. Bundesgesundheitsblatt Gesundheitsforschung Gesundheitsschutz 52(8):874-877. doi:10.1007/ s00103-009-0902-9

16. Wiesmüller GA, Eckard R, Dobler L, Günsel A, Oganowski M, Schröter-Kermani C, Schlüter C, Gies A, Kemper FH (2007) The environmental specimen bank for human tissues as part of the german environmental specimen bank. Int J Hyg Environ Health 210:299-305

17. Dobler L, Eckard R, Günsel A, Müller A, Oganowski M (2011) Umweltprobenbank des Bundes - Teilbank Humanproben und Datenbank - Vergleich der Schadstoffbelastung bei Studierenden in den neuen und alten Bundesländern zwischen 1997 und 2009. Projektbericht Westfälische Wilhelms-Universität Münster. http://www. umweltprobenbank.de/upb_static/fck/download/ Bericht_West_Ost_FINAL2.pdf. Zugegriffen: 22. Juni 2016 
18. Dobler L, Eckard R, Günsel AK, Langel D, Müller A, Oganowski M, Wiesmüller GA (2011) Umweltprobenbank des Bundes - Teilbank Humanproben und Datenbank -Jahresbericht 2010/11, Westfälische Wilhelms-Universität Münster. http:// www.umweltprobenbank.de/upb_static/fck/ download/Jahresbericht_Human_2010_2011.pdf. Zugegriffen: 22. Juni 2016

19. Grandjean P, Budtz-Jorgensen E, Barr DB, Needham LL, Weihe P, Heinzow B (2008) Elimination half-lives of polychlorinated biphenyl congeners in children. Environ Sci Technol 42(18):6991-6996

20. Ritter R, Scheringer M, MacLeod M, Schenker U, Hungerbühler K (2009) A multi-individual pharmacokinetic model framework for interpreting time trends of persistent chemicals in human populations: application to a postban situation. Environ Health Perspect 117(8):1280-1286

21. Quinn CL, Wania F, Czub G, Breivik K (2011) Investigating intergenerational differences in human PCB exposure due to variable emissions and reproductive behaviors. Environ Health Perspect 119:641-646

22. Quinn CL, Wania F (2012) Understanding differences in the body burden - age relationships of bioaccumulating contaminants based on population cross sections versus individuals. Environ Health Perspect 120:554-559

23. Schettgen T, Gube M, Alt A, Fromme H, Kraus $T$ (2011) Pilot study on the exposure of the general population in Germany to non-dioxin-like and dioxin-like PCBs. Int J Hyg Environ Health 214(4):319-325

24. Fromme H, Albrecht $M$, Appel M, Hilger B, Völkel W, Liebl B, Roscher E (2015) PCBs, PCDD/Fs, and PBDEs in blood samples of a rural population in South Germany. Int J Hyg Environ Health 218(1):41-46

25. Schettgen T, Alt A, Esser A, Kraus T (2015) Current data on the background burden to the persistent organochlorine pollutants $\mathrm{HCB}, \mathrm{p}, \mathrm{p}^{\prime}-\mathrm{DDE}$ as well as PCB 138, PCB 153 and PCB 180 in plasma of the general population in Germany. Int J Hyg Environ Health 218:380-385

26. Kommission Human-Biomonitoring des Umweltbundesamtes (2012) Human-Biomonitoring (HBM)-Werte für Polychlorierte Biphenyle (PCB) im Blut. Bundesgesundheitsblatt Gesundheitsforschung Gesundheitsschutz 55(8):1069-1070

27. Hardell E, Carlberg M, Nordström M, van Bavel $B$ (2010) Time trends of persistent organic pollutants in Sweden during 1993-2007 and relation to age, gender, body mass index, breast-feeding and parity. Sci Total Environ 408(20):4412-4419

28. Ritter R, Scheringer M, MacLeod M, Schenker U, Hungerbühler K (2009) A multi-individual pharmacokinetic model framework for interpreting time trends of persistent chemicals in human populations: application to a postban situation. Environ Health Perspect 117(8):1280-1286

29. Ritter R, Scheringer M, MacLeod M, Moeckel C, Jones KC, Hungerbühler K (2011) Intrinsic human elimination half-lives of polychlorinated biphenyls derived from the temporal evolution of cross-sectional biomonitoring data from the United Kingdom. Environ Health Perspect 119(2):225-231 